\title{
REVISITED HISTOGRAM EQUALIZATION AS HDR IMAGES TONE MAPPING OPERATORS
}

\author{
Anas Husseis ${ }^{1}$, Anissa Mokraoui ${ }^{1}$, Basarab Matei ${ }^{2}$ \\ ${ }^{1}$ L2TI, ${ }^{2}$ LIPN, Institut Galilée, Université Paris 13 Sorbonne Paris Cité \\ 99 avenue Jean-Baptiste Clément, 93430 Villetaneuse, France \\ anas.husseis@telecom-paristech.fr, anissa.mokraoui@univ-paris13.fr, matei@lipn.univ-paris13.fr
}

\begin{abstract}
High Dynamic Range (HDR) image provides higher perceptual quality such that it appears considerably more realistic and attractive for the human observer. Since most of current screens are Low Dynamic Range (LDR) screens, lots of researches have been proposed to design tone mapping algorithms converting the HDR images into a range that is suitable to display these tone mapped images on standard LDR screens. For this purpose, this paper first investigates the pixels distribution in the HDR image through the study of various traditional Histogram Equalization (HE) algorithms which were originally developed to improve the visual quality of LDR images such as the contrast enhancement of the latter. Moreover, a modification of the Histogram Adjustment based Linear to Equalized Quantizer (HALEQ), developed for HDR images, is proposed. Simulation results show that the proposed modification preserves more details than the original version of the algorithm in most parts of the HDR image.
\end{abstract}

\section{INTRODUCTION}

High Dynamic Range (HDR) images consists of pixels, which contains greater range of colors and brightness levels than the range of Low Dynamic Range (LDR) images. Therefore displaying the HDR images on standard LDR screens remains a challenging problem. Indeed reducing the high into low dynamic range relies on a lossy transformation. This latter is known as Tone Mapping (TM) operation. Recently, significant number of Tone Mapping Operators (TMOs) converting the HDR images to LDR images have been proposed using different strategies. Among the developed methods, some of them were designed with respect to the Human Visual System (HVS) [1], another were based on the frequency domain [2]-[4], segmentation operators were also designed to combine multiple functions to different segments [13], [14]. However, tone mapped images suffer from data loss because of the reduction process into low dynamic range, affecting the quality of the tone mapped images.

This paper focuses on tone mapping operators that consider both Histogram Equalization (HE) and human sensitivity to the light function. To do so, a logarithmic transform is first applied on the HDR luminance to produce a distribution that is close to the HVS [11], [12]. The target then is to redistribute this logarithmic luminance on a given number of intervals corresponding to the standard LDR display such that it is equally distributed on each interval. This ensures the randomness maximization on the redistributed probability density function (pdf) respecting the human sensitivity to the light.

Several well known HE techniques have been deployed to enhance the LDR image contrast by redistributing the pdf of the pixels to obtain a flat histogram. This paper first investigates these traditional HE methods to render the HDR images at LDR displays to not only preserve the contrast and brightness but also achieve maximum randomness in the tone mapped images. Then, a modification is made to the Histogram Ajustement Linear to Equalization algorithm (HALEQ) dedicated for tonemapped HDR images [10].

The rest of this paper is organized as follows. Next section revisits the traditional histogram equalization methods, originally developed to improve the visual quality of LDR images, to be considered as TMO for HDR images. Section 3 presents the generic Histogram Adjustment based Linear to Equalized Quantizer (HALEQ) TMO. A modification is then made to improve its performance in terms of objective and subjective quality. Section 4 analyses and discusses the simulation results. Section 5 concludes this paper.

\section{REVISITED HISTOGRAM EQUALIZATION AS HDR IMAGE TMO}

This section concerns HE methods to map HDR into LDR images while preserving the most important details in the HDR images in accordance to the range of the LDR device. These methods are derived from those initially deployed for LDR images to enhance their visual quality. These techniques, considered in this paper as TMOs, aim to produce images with an applicable dynamic range on the standard LDR display. Note that the probability density function of the HDR pixel values usually behaves in Laplacian manner and manipulating the linear values of luminance causes heavy distortion because of the insignificant relation to the visual perception [6]. Weber law can 
be used to conclude a straightforward relationship between the sensitivity function and the logarithmic transform of the luminance. Therefore to take into account the human sensitivity function to light, the derived HE techniques will operate on the logarithmic luminance of the HDR images.

A HE technique is a nonlinear operation that modifies, in an appropriate way, the initial distribution (i.e. pdf) of the pixels in the HDR image to produce a uniform distribution (i.e. a flat histogram) over the range of the LDR display. The mathematical explanation behind this strategy is based on the entropy of the source. Consider a source of logarithmic luminance events $\left\{x_{1}, \ldots, x_{n}\right\}$ with their probabilities $\left\{p\left(x_{1}\right), \ldots, p\left(x_{n}\right)\right\}$ (i.e. the source pdf). The entropy, denoted $H(x)$, is given by the average amount of information as follows [5] :

$$
H(x)=-\sum_{i=1}^{n} p\left(x_{i}\right) \log \left(p\left(x_{i}\right)\right) .
$$

According to this definition, the entropy is maximized when the events (logarithmic luminance levels) are uniformly distributed. Therefore applying $\mathrm{HE}$ operation to the source ensures that maximum possible amount of information is contained in the tone-mapped image.

Therefore this paper exploits HE techniques to map the logarithmic luminance from HDR into LDR such that the reduced range corresponds to the LDR display, taking into account the pixels population distribution. In the following, four HE methods are investigated : starting with Global Histogram Equalization (GHE), continuing with HE development in order to maximize the brightness preservation using Brightness Preserving Bi-Histogram Equalization (BBHE) [7], Dualistic Sub-Image Histogram Equalization (DSIHE) [8], and Recursive Mean-Separate Histogram Equalization (RMSHE) [9].

Before presenting these techniques, let us introduce some notations. Assume that the HDR image is of size $N=n \times m$ pixels. The HE divides the logarithmic luminance HDR into $K$ intervals in accordance to the bit depth of the LDR screen, such that each interval contains $\frac{n \times m}{K}$ pixels. Denote $L_{w}(i, j)$ the logarithmic luminance of the pixel at position $(i, j)$ on the HDR image. Denote $L_{w_{m i n}}$ (respectively $L_{w_{\max }}$ ) the smallest (respectively highest) logarithmic luminance of the HDR image.

\subsection{Global Histogram Equalization}

Global Histogram Equalization (GHE) maps the logarithmic luminance HDR into LDR of $K$ levels such that all reproduced levels have equal amount of pixels. GHE objective is to produce a luminance that takes values in the sub-intervals $\left[D_{k}, D_{k+1}\right]$ such that $L_{d}(i, j) \in$ $\left\{D_{0}, D_{1}, \ldots, D_{K-1}\right\}$.

The probability density function (pdf) according to the previous model is formulated as follows :

$$
p\left(D_{k}\right)=\frac{N_{k}}{N}
$$

where $D_{k}$ is the display level, $N_{k}$ is the pixel population at each interval $\left[D_{k}, D_{k+1}\right]$ and $N$ is the total amount of pixels in the image.

The cumulative distribution function (cdf) of $D_{k}$ is given by :

$$
C\left(D_{k}\right)=\sum_{j=0}^{k} p\left(D_{k}\right)=\sum_{j=0}^{k} \frac{N_{k}}{N}, k \in[0, K-1] .
$$

$C\left(D_{k}\right)$ is a non-decreasing function because it is the empirical probability distribution function and it takes the maximum value at the last point such that $C\left(D_{K-1}\right)=1$.

To equalize and map the histogram, primarily the discrete cumulative normalized histogram $C\left(D_{k}\right)$ has to be calculated and applied at each logarithmic luminance value according to the following formula :

$L_{d}(i, j)=f\left(L_{w}(i, j)\right)=D_{0}+\left(D_{K-1}-D_{0}\right) C\left(L_{w}(i, j)\right)$,

where $L_{w}(i, j)$ is the logarithmic luminance value and $C\left(L_{w}(i, j)\right)$ is its cumulative probability which considers $K$ intervals corresponding to the LDR screen. The display luminance $L_{d}$ has approximately a flat histogram not a perfectly flatted one, because histogram bins are never decreased in amplitude, though they may grow if various luminance levels map to the same value.

GHE produces $L_{d}$ logarithmic luminance values which are suitable with LDR displays, taking into account pixel population distribution. The main drawback of GHE is that the mean value of $L_{d}$ does not take into account the mean value of $L_{w}$. In other words, the brightness of the HDR image is not preserved in the tone mapped image. Several techniques were then proposed to bypass this drawback as it will be discussed in the next sections.

\subsection{Brightness Preserving Bi-Histogram Equalization}

Brightness Preserving Bi-Histogram Equalization (BBHE) technique is an intuitive technique, originally used to preserve the brightness of the enhanced LDR images [7]. This approach is exploited as a tone mapping operator. It consists of cutting the logarithmic luminance HDR histogram into two sub-intervals using a selected threshold $L_{T}$. This latter is deduced as the average of the logarithmic luminance values $\left(\left[L_{w_{\text {min }}}, L_{w_{\text {max }}}\right]\right)$ of the HDR image. The GHE strategy, described in section 2.1, is then applied on each sub-interval (i.e. $\left[L_{w, \min }, L_{T}\right]$ and $\left.\left[L_{T}, L_{w, \max }\right]\right)$. This separation into two sub-intervals grants the preservation of the mean brightness of the HDR image.

\subsection{Dualistic Sub-Image Histogram Equalization}

Dualistic Sub-Image Histogram Equalization (DSHE) technique has been developed to enhance the visual quality of LDR images [8]. In this section, it is exploited as a TMO for HDR images. The original logarithmic luminance of the HDR image is decomposed into two sub-intervals 
where each of them contains the half amount of the total number of pixels of the HDR image. This proposition is based on Shannon entropy function :

$$
h=-p \log (p)-(1-p) \log (1-p),
$$

where $\mathrm{p}$ corresponds to the probability distribution of the first interval. The entropy of the aggregation dualistic segmentation is maximized when $p=0.5$, consequently $(1-p)$ represents the probability distribution of the other interval. In order to satisfy this assumption, $L_{T}$ is defined as the median of $L_{w}$. Then each interval is equalized using the GHE strategy (see section 2.1). In this technique, significant shifts on the average logarithmic luminance is avoided especially for the large regions with same luminance level.

\subsection{Recursive Mean-Separate Histogram Equalization}

Recursive Mean-Separate Histogram Equalization (RMSHE) technique has been developed for better brightness preserving of the LDR images [9]. It is exploited in this paper as a TMO for HDR images. Instead of applying a single cut, as in BBHE, a recursive process takes place to cut in an iterative way the sub-intervals according to different cutting points $\left\{L_{T_{1}}, L_{T_{2}}, \ldots, L_{T_{N_{I-1}}}\right\}$ (with $r$ the number of iterations that produces $N_{I}=2^{r}$ subintervals) deduced as in section 2.2. The GHE technique is applied at each sub-interval as discussed before. Note that choosing $r=0$ is equivalent to GHE, while $r=1$ corresponds to BBHE. So this method provides a scalable brightness preservation higher than that in BBHE and DSIHE while choosing $r \geqslant 2$.

\section{MODIFIED HISTOGRAM ADJUSTMENT BASED LINEAR TO EQUALIZED QUANTIZER}

Before presenting the modification made to the Histogram Adjustment based Linear to Equalized Quantizer (HALEQ), developed in [10], this section starts with the description of the generic algorithm.

HALEQ is a hybrid TMO that considers both HE and linear mapping (uniform quantization) while reducing the high dynamic range. The logarithmic transform is applied to the world luminance as a first step through the following expression [11], [12] :

$$
\begin{aligned}
& D(I)=\left(D_{\max }-D_{\min }\right) \\
& \times \frac{\log (I+\tau)-\log \left(I_{\min }+\tau\right)}{\log \left(I_{\max }+\tau\right)-\log \left(I_{\min }+\tau\right)}+D_{\min },
\end{aligned}
$$

where $I$ is the world luminance ; $D_{\min }$ and $D_{\max }$ are the minimum and maximum level values for the LDR display device (typically they are $[0,255]) ; I_{\min }$ and $I_{\max }$ are extracted from the HDR image to represent the lowest and highest luminance values in the scene ; and $\tau$ is a constant which controls the overall brightness of the tone mapped image.

The tone mapping operation is performed in a recursive algorithm such that at the first iteration (i.e. $r=1$ ) the dynamic range is divided twice : (i) it is divided into two equal sub-intervals using the cutting point $l_{n}$ (linear mapping); and (ii) it is divided into two sub-intervals, using the cutting point $e_{n}$, in order to get the same number of pixels in each interval (HE). An adjustment process is then performed by choosing a new cutting point $l_{e n}$ between these two cutting points according to the following relationship :

$$
l_{e n}=l_{n}+\beta\left(e_{n}-l_{n}\right),
$$

where $\beta$ is a fixed constant taking its value between 0 and 1.

This new cutting point $l_{e n}$ is controlled by the parameter $\beta$ which is fixed once by the authors at the beginning of the process. The described process is then iterated. Indeed at each iteration the dynamic range of the sub-intervals are cut once again to produce $2^{r}$ sub-intervals where $r$ is the number of iterations corresponding to the capability of the LDR screen.

In order to improve, in particular, the visual quality of the tone mapped HDR image, this section proposes to adapt the value of $\beta$ to each sub-intervals rather than using the same constant value for all sub-intervals (see equation (7)). Equation (7) becomes :

$$
l_{e n}=l_{n}+\beta_{n}\left(e_{n}-l_{n}\right),
$$

where $\beta_{n}$ is a parameter depending on the sub-interval $\left[I_{n}, e_{n}\right]$.

Moreover, we propose to compute the cutting point $l_{e n}$ as the average luminance on the sub-interval $\left[I_{n}, e_{n}\right]$. From equation (8), the $\beta_{n}$ is then deduced as follows :

$$
\beta_{n}=\frac{\bar{D}\left(I_{e_{n}, l_{n}}\right)-l_{n}}{e_{n}-l_{n}},
$$

where $\bar{D}\left(I_{e_{n}, l_{n}}\right)$ is the average logarithmic luminance in the sub-interval $\left[e_{n}, l_{n}\right]$.

This strategy provides an automatic, simple and efficient way to assign the value $\beta_{n}$ at each cut such that the brightness preservation is maximized in the tone mapped images.

\section{SIMULATION RESULTS}

This section evaluates the performance of the revisited and modified histogram equalization methods that have been presented in the previous sections. Simulations have been conducted under Matlab environnement using the HDR Toolbox with its HDR test images [16]. HE methods are tested on 10 indoor and outdoor HDR images. An objective performance evaluation based on TMQI (ToneMapped image Quality Index) metric, that considers the Structural SIMilarity and the Structural Naturalness between the tone mapped image and the HDR image [15], has been performed. This metric is upper-bounded by 1 .

Table 1 provides the TMQI of the tone mapped HDR images according to the revisited HE methods using 8 bits 
of color precision per R,G,B color channel. The TMQI metric values are close to 1 (ranging between 0.88 and 0.97). Subjective visual quality of these tone mapped images are also analyzed. Fig. 1, Fig. 2, Fig. 3 and Fig. 4 show the "Clock Building" and "Bottles" tone mapped HDR images. One can observe the excellent contrast and the preserved details on the mapped images. Indeed the fine details on the sky for "Clock Building" image and on the wall for the "Bottles" image are very well rendered. Over and above, the brightness preservation is maximized on the RMSHE as we assumed $r=2$ to obtain 4 sub-intervals providing pleasing images with high TMQI values.

\begin{tabular}{lcllc}
\hline TMOs & GHE & BBHE & DSIHE & RMSHE \\
\hline Bottles & 0.913 & 0.894 & 0.901 & 0.888 \\
Clock Building & 0.957 & 0.970 & 0.970 & 0.957 \\
Office & 0.955 & 0.933 & 0.949 & 0.875 \\
Apartment Backdrop & 0.937 & 0.943 & 0.945 & 0.904 \\
Atrium Night & 0.912 & 0.842 & 0.842 & 0.808 \\
Belgium & 0.948 & 0.942 & 0.935 & 0.872 \\
Doll & 0.885 & 0.891 & 0.891 & 0.848 \\
Memorial Church & 0.904 & 0.888 & 0.887 & 0.886 \\
Oxford Church & 0.968 & 0.972 & 0.967 & 0.942 \\
Small Office & 0.959 & 0.965 & 0.967 & 0.940 \\
\hline
\end{tabular}

Table 1. TMQI of the revisited HE methods.

On the other hand, the optimized HALEQ TMO is also tested. Table 2 compares the TMQI metric according to the values of $\beta$ considered as a constant or an optimized value. The visual quality of the tone mapped images are also compared when using a constant $\beta$ value (e.g. $\beta=0.5$ ) during all the process and an optimized value (see Fig. 5 and Fig. 6). One can observe that fine details are better rendered on the optimized $\beta$. This can be obviously noticed from the sky on "Clock Building" image and the wall on "Bottles" image. This is explained by the fact that $\beta$ is well adapted to the different sub-intervals. Fig. 7 (respectively Fig. 8) shows the different values of $\beta$ that have been used by the optimized HALEQ TMO for the "Clock Building" (respectively "Bottles") HDR image. The sensitivity curve given by Fig. 12, for "Clock Building", shows that the curve for $\beta=0.75$ is close to the curve with optimal $\beta$ that is why the TQMI metric are almost equal.

Fig. 9 illustrates the pdf of the luminance "Clock Building" HDR image. The normalized histograms of the equalized HDR image according respectively to the generic and modified HALEQ are provided by Fig. 10 (with $\beta=0.5$ and a standard deviation $\sigma=0.0040$ ) and Fig. 11 (with a standard deviation $\sigma=0.0033$ ). The modified HALEQ histogram is flatter than that obtained by the generic HALEQ.

Fig. 12 provides the histogram of the equalized "Clock Building" HDR image with $\beta=0.75$ and a standard deviation $\sigma=0.0031$. The latter is equivalent to that of the modified HALEQ algorithm. That is why the TMQI metrics are similar (0.96 see Table 2$)$.

\section{CONCLUSION}

This paper revisited several histogram equalization techniques that have been essentially proposed to enhance
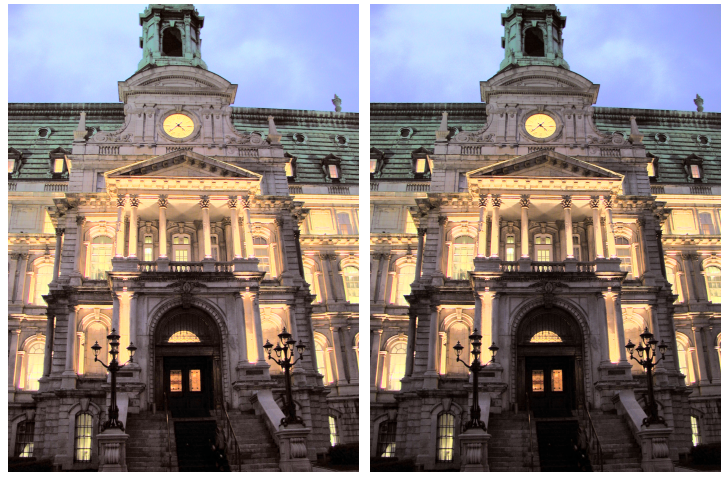

Fig. 1. GHE (left image); BBHE (right image) using "Clock Building" HDR image.
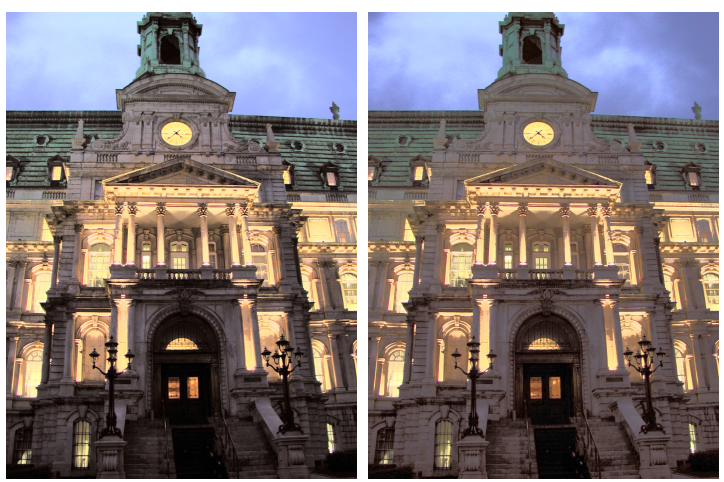

Fig. 2. DSIHE (left image); RMSHE (right image) using "Clock Building" HDR image.
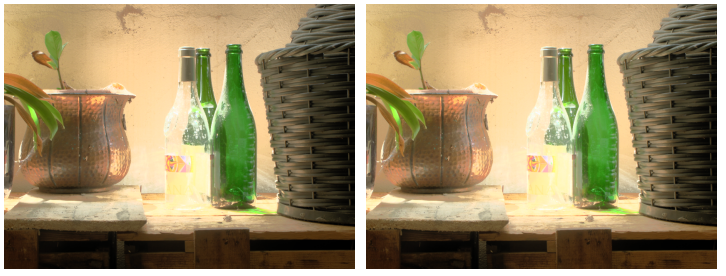

Fig. 3. GHE (left image); BBHE (right image) using "Bottles" HDR image.
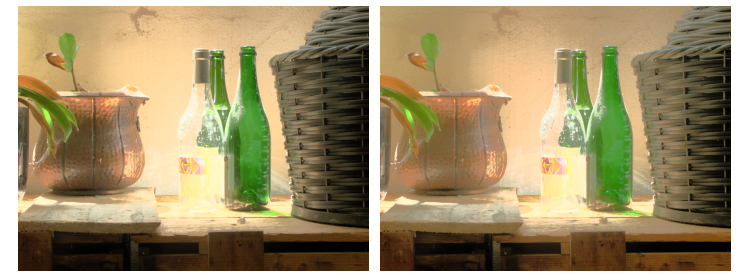

Fig. 4. DSIH (left image); RMSHE (right image) using "Bottles" HDR image.

the contrast of LDR images. These HE methods have been applied on HDR images as TMO to achieve a limited 

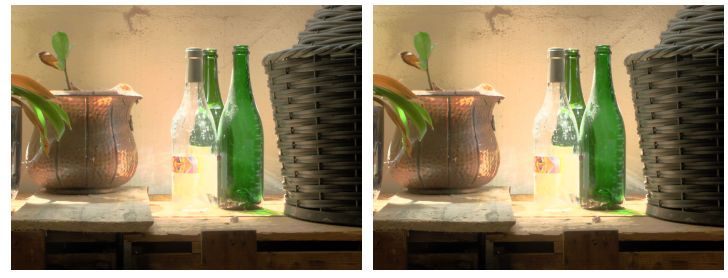

Fig. 5. HALEQ with $\beta=0.5$ (left image); HALEQ with optimized $\beta$ (right image) using "Bottles" HDR image.
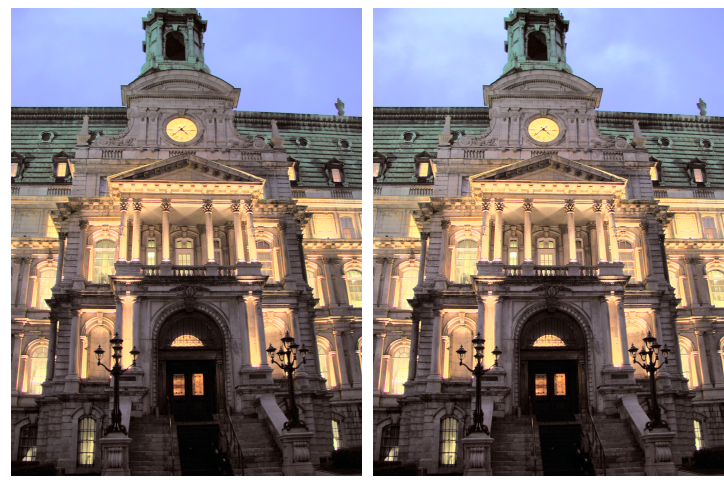

Fig. 6. HALEQ with $\beta=0.5$ (left image); HALEQ with optimized $\beta$ (right image) using "Clock Building" HDR image.

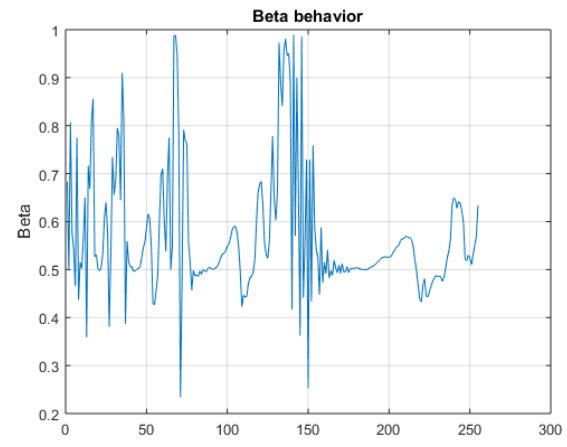

Fig. 7. Optimized $\beta$ for "Clock Building" HDR image.

\begin{tabular}{lcccc}
\hline HALEQ TMO & $\beta=0.25$ & $\beta=0.5$ & Optimized $\beta$ & $\beta=0.75$ \\
\hline Bottles & 0.870 & 0.915 & 0.916 & 0.920 \\
Clock Building & 0.870 & 0.951 & 0.961 & 0.965 \\
Office & 0.867 & 0.968 & 0.963 & 0.970 \\
Apartment Backdrop & 0.839 & 0.929 & 0.940 & 0.941 \\
Atrium Night & 0.942 & 0.964 & 0.933 & 0.944 \\
Belgium & 0.905 & 0.961 & 0.958 & 0.962 \\
Doll & 0.821 & 0.882 & 0.887 & 0.888 \\
Memorial Church & 0.846 & 0.935 & 0.915 & 0.918 \\
Oxford Church & 0.892 & 0.985 & 0.980 & 0.984 \\
Small Office & 0.898 & 0.955 & 0.961 & 0.965 \\
\hline
\end{tabular}

Table 2. TMQI of HALEQ and modified HALEQ methods.

dynamic range adapted to LDR screens. These techniques are not only simple and easy to implement but also provide good performance both in terms of objective and subjective

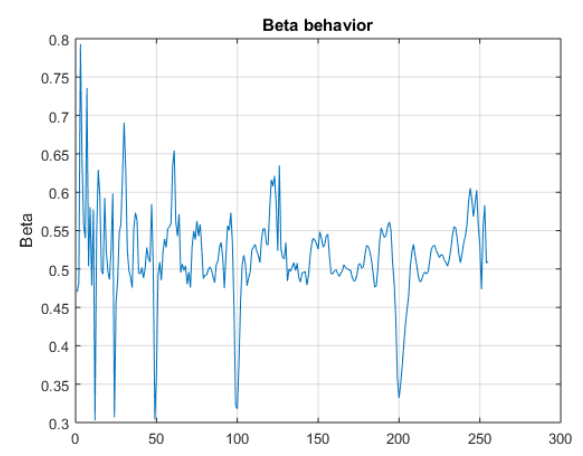

Fig. 8. Optimized $\beta$ for "Bottles" HDR image.

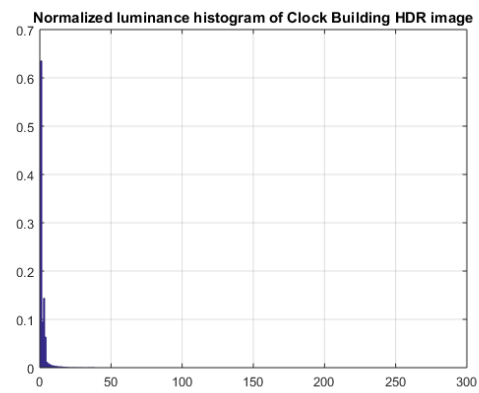

Fig. 9. "Clock Building" HDR image histogram.

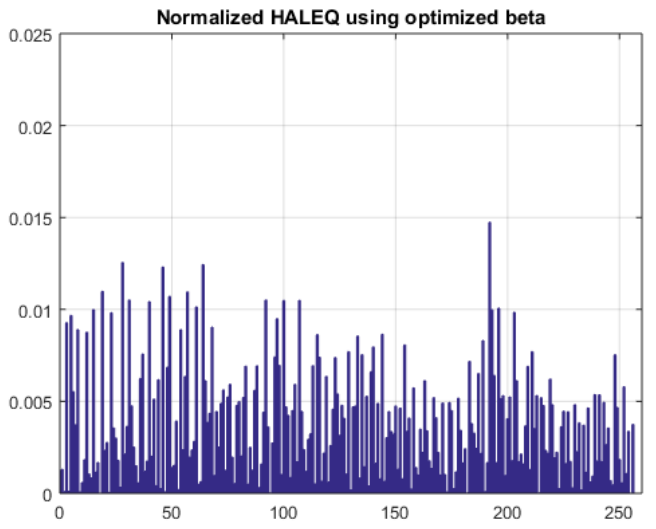

Fig. 10. "Clock Building" tone mapped image histogram using the modified HALEQ (optimized $\beta$ ).

quality. Moreover, a modification has been made to the generic HALEQ tone mapping operator such that the parameter $\beta$ is automatically adjusted according to the pdf of the HDR image. Simultation results show that this modification preserves the brightness and details of the HDR image by optimizing the cutting points between the linear mapping and HE. 


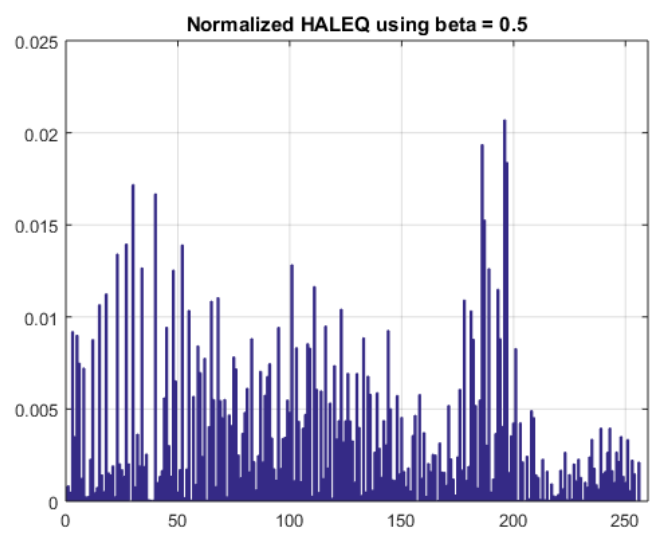

Fig. 11. "Clock Building" tone mapped image histogram using HALEQ with $\beta=0.5$.

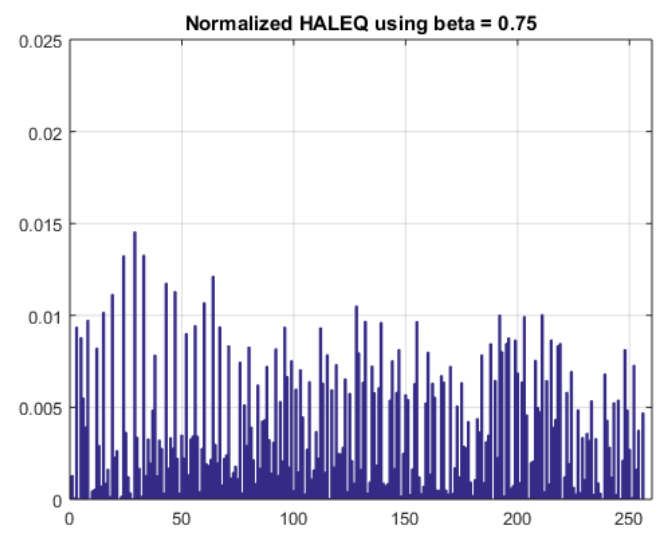

Fig. 12. "Clock Building" tone mapped image histogram using HALEQ with $\beta=0.75$.

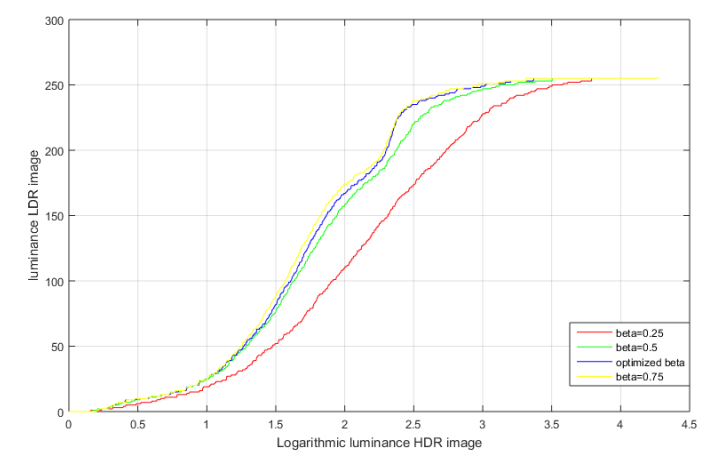

Fig. 13. "Clock Building" LDR versus HDR logarithmic luminance using HALEQ and modified HALEQ.

\section{REFERENCES}

[1] Jack Tumblin, Jessica K. Hodgins, and Brian K. Guenter. Two Methods for Display of High Contrast Images. ACM
Trans. Graph. 18 :1, pp. 56-94 (1999).

[2] Jack Tumblin and Greg Turk. LCIS : A Boundary Hierarchy for Detail Preserving Contrast Reduction. In SIGGRAPH : Proceedings of the 26th Annual Conference on Computer Graphics and Interactive Techniques, pp. 8390. New York, NY, USA : ACM Press/Addison-Wesley Publishing Co., (1999).

[3] Raanan Fattal, Dani Lischinski, and Michael Werman. Gradient Domain High Dynamic Range Compression. ACM Trans. Graph. 21 :3, pp. 249-256, (2002).

[4] Thai, B.C., Mokraoui, A., and Matei, B., Performance Evaluation of High Dynamic Range Image Tone Mapping Operators Based on Separable Non-linear Multiresolution Families. 24th European Signal Processing Conference, pp. 1891-1895 (August 2016).

[5] C. Shannon, A mathematical theory of communication. Bell Syst. Tech. J., vol. 27, 379423, (1948).

[6] A. Boschetti, N. Adami, R. Leonardi and M. Okuda, High Dynamic Range image tone mapping based on local Histogram Equalization. 2010 IEEE International Conference on Multimedia and Expo, Suntec City, pp. 1130-1135, (2010).

[7] Yeong-Taeg Kim, Contrast enhancement using brightness preserving Bi-Histogram equalization. IEEE Trans. Consumer Electronics, vol. 43, no. 1, pp. 1-8, Feb. (1997).

[8] Yu Wang, Qian Chen and Baeomin Zhang, Image enhancement based on equal area dualistic sub-image histogram equalization method. In IEEE Transactions on Consumer Electronics, vol. 45, no. 1, pp. 68-75, Feb (1999).

[9] S.-D. Chen, A.R. Ramli, Contrast enhancement using recursive mean separate histogram equalization for scalable brightness preservation. IEEE Trans. Consum. Electron, 49 pp. 1301-1309, (2003).

[10] Jiang Duan, Marco Bressan, Chris Dance, Guoping Qiu. Tone-mapping high dynamic range images by novel histogram adjustment. Pattern Recognition 43, pp. 1847-1862, (2010).

[11] F. Drago, K. Myszkowski, T. Annen, N. Chiba, Adaptive logarithmic mapping for displaying high contrast scenes. The Journal of Computer Graphics Forum 22 (3), pp. 419426, (2003).

[12] T.G. Stockham, Image processing in the context of a visual model. Proceedings of the IEEE 60 (7), pp. 828-842, (1972).

[13] Dani Lischinski, Zeev Farbman, Matt Uyttendaele, and Richard Szeliski. Interactive Local Adjustment of Tonal Values. ACM Trans. Graph. 25 :3, pp. 646-653, (2006).

[14] Grzegorz Krawczyk, Karol Myszkowski, and Hans-Peter Seidel. Lightness Perception in Tone Reproduction for High Dynamic Range Images. In The European Association for Computer Graphics 26th Annual Conference EUROGRAPHICS, pp. 635-645. Dublin, Ireland : Blackwell, (2005).

[15] H. Yeganeh and Z. Wang, Objective Quality Assessment of Tone-Mapped Images. In IEEE Transactions on Image Processing. Vol. 22, no. 2, pp. 657-667, Feb. (2013).

[16] Banterle, F., Artusi, A., Debattista, K., and Chalmers, A., Advanced High Dynamic Range Imaging : Theory and Practice. AK Peters (now CRC Press), ISBN : 9781568817194 (2011). 